\title{
Why can't Chinese Han drink alcohol? Hepatitis B virus infection and the evolution of acetaldehyde dehydrogenase deficiency
}

\author{
Y. -P. Lin, ${ }^{1}$ T. -J. Cheng ${ }^{2}$ \\ ${ }^{1}$ Institute of Health Policy and Management, College of Public Health, National Taiwan University, Taipei, Taiwan; ${ }^{2}$ Institute of Occupational \\ Medicine and Industrial Hygiene, College of Public Health, National Taiwan University, Taipei, Taiwan
}

Summary An aldehyde-dehydrogenase (ALDH2) deficiency is a biological curb on excess alcohol-drinking. This enzyme deficiency is very common amongst Oriental people while it is relatively rare for most other populations. We observe that there is good geographical correlation between the prevalence of the mutant ALDH2* 2 alleles and hepatitis $B$ virus (HBV) infections. Populations that demonstrate a high ALDH2 2 prevalence are all located in HBV-endemic areas. Further, studies have shown that HBV and alcohol drinking exhibit a synergistic effect upon liver cirrhosis and cancer. A shorter life span for those with HBV infection and heavy alcohol consumption may result in a selection of the ALDH2*2 gene. We postulate that there may be patterns of evolutionary adaptation for ALDH2 deficiency in certain HBV-endemic areas and that these adaptations can produce differences in human alcohol-drinking capability. () 2002 Elsevier Science Ltd. All rights reserved.

\section{INTRODUCTION}

Chinese people have a long history of drinking alcoholic beverages, textual accounts and archeological remains of drinking utensils point to the indulgence of alcohol by Shang noblemen (18-12th century BC) (1). Since the earliest of times, alcohol drinking has been a basic part of Chinese living, whether in the highest rituals or in any casual moment of relaxation by the humblest member of society (2). The history of China includes several abortive efforts to control the preparation and consumption of alcoholic beverages or even their prohibition. It has

Received 24 August 2001

Accepted 19 December 2001

Correspondence to: Dr. Tsun-Jen Cheng, Institute of Occupational Medicine and Industrial Hygiene, 1 Ren-Ai Road Section 1, Taipei, Taiwan, 100.

Phone \& Fax: +886-2-2395-7845;

E-mail: tcheng@ha.mc.ntu.edu.tw been said that Xia-Yu, the first emperor of the Xia dynasty (2183-1752 BC), expelled the royal winemaker out of the fear that his overly indulgent descendants might end the empire.

Peculiarly, modern Chinese society seems to demonstrate only minor alcohol-drinking problems (3).

\section{ALCOHOL CONSUMPTION AND ALDH2 GENES}

Epidemiological studies have reported that Asians are less likely to become alcohol-dependent compared to other ethnic groups $(4,5)$. Approximately half of Chinese, Japanese, and Korean people exhibit a deficiency in aldehyde dehydrogenase (ALDH2), which is responsible for metabolizing acetaldehyde into acetic acid, a component of the alcohol-metabolizing human biochemical pathway (6). A deficiency in ALDH2 activity may result from the inheritance of the mutant ALDH2*2 allele, a dominant mutation that exerts its effect both by reducing the enzyme's activity and also by increasing 
the turnover of this enzyme (7). After the ingestion of alcohol, the faces of Asians expressing one or both alleles of ALDH2*2 become visibly flushed (known as 'oriental flush') (8). An increased blood acetaldehyde level following the consumption of alcohol by Asians with one or both alleles of ALDH2*2 expressed also causes tachycardia, nausea and other unpleasant symptoms (7). Polymorphisms of ALDH2 genes modify alcohol flushing, alcohol-drinking behavior and also the potential risk for alcoholism $(8,9)$.

\section{THE DISTRIBUTION OF ALDH2 GENES}

There are wide ethnic variations in the distribution of the ALDH2*2 allele. This allele is very common amongst Asian persons and appears to be rare for most other populations (7). Thirty to fifty percent of Orientals, including Chinese, Japanese, Koreans, Filipinos, Thais, and Vietnamese, are deficient in ALDH2 activity, while a negligible fraction of persons in Africa and Europe are similarly deficient. Further, approximately $40 \%$ of South American Indians in Chile and Ecuador are deficient in ALDH2 activity, but only $2-5 \%$ of North American and Mexican Indians are deficient in this enzyme. Amongst Chinese ethnic groups, the Han sub-population reveals the highest percentage of ALDH2 deficiency (45\%) as compared to other minority groups (e.g. 30\% of Mongolian, 25\% of Zhuang, 25\% of Mandschu, and a negligible portion of Taiwanese aborigines) (10-12).

Moderate alcohol-drinking in certain populations, however, can be more beneficial than hazardous. Certain epidemiological studies have indicated that the regular consumption of small amounts of alcohol is associated with lower overall mortality $(13,14)$. From the wide geographical variations of ALDH2*2 allele distribution, we speculate that the genetic factors favoring non-alcohol-drinking in certain geographical areas may be associated with liver diseases.

\section{LIVER DISEASES AND HEPATITIS B VIRUS INFECTION}

In Taiwan, cancer has been the leading cause of death since 1982, and liver cancer is the most common cause of male cancer death (15). Further, chronic liver disease and liver cirrhosis is the fifth leading cause of death in Taiwan (15). The high prevalence of cirrhosis and liver cancer in Taiwan is attributed to the high prevalence of infection by the hepatitis B virus (HBV) $(16,17)$. Most HBV carriers in Taiwan were infected by perinatal transmission from their mothers or by horizontal transmission in their childhood (18). Cases of HBV-related liver cancer may occur as early as from six to 14 years of age (19).
Beasley and colleagues' 1982 study of the HBV carrier rates by Chinese province of origin suggested that HBV carrier rates were greatest in southeastern and least in northwestern China (20). Sung and Chen (1977) also observed that men in Taiwan originating from north of the Yangtze River revealed different HBV subtypes compared to those deriving from south of the river (21). In 1982, Beasley and colleagues proposed that the endemic HBV revealed a long history in China, and that the virus was carried south during the Han dynasty (206 $\mathrm{BC}-221 \mathrm{AD}$ ) essentially through the migration of family units (20).

Globally, the geographical areas at highest risk of liver cancer and HBV prevalence are located in Eastern Asia and Africa, and those geographical areas at lowest risk are North Europe, Australia, New Zealand, and the Caucasian populations of North and Latin America (22).

\section{THE INTERACTION BETWEEN ALCOHOL- DRINKING AND HBV INFECTION}

Alcohol drinking, cigarette smoking, and aflatoxin are risk factors that synergistically interact with $\mathrm{HBV}$ in the development of liver cancer (23). Currently, however, the detailed mechanism of such effects remains unclear.

In a case-control study in Italy, a strong interaction between HBV positivity and heavy alcohol intake was found, the study suggesting the more-than-additive effects of viral infections and alcohol-drinking upon the risk of hepatocellular carcinoma (24). From this Italian study, the relative risk of hepatocellular carcinoma was estimated to be 64.7 for concurrent HBV and heavy alcohol intake (more than $80 \mathrm{~g}$ per day for at least 5 years), and 9.1 for concurrent HBV with a lower alcohol intake. In this Italian study, $58.1 \%$ of cases of hepatocellular carcinoma and $36.4 \%$ of controls were defined as individuals reflecting a heavy alcohol intake.

Nevertheless, in Yu and colleagues' 1997 prospective study of hepatocellular carcinoma and liver cirrhosis amongst asymptomatic chronic HBV carriers in Taiwan, habitual alcohol drinking was not independently related to liver cirrhosis (25). Although there were $21 \%$ of the $1506 \mathrm{HBV}$ carriers being defined as 'habitual alcohol drinking' in this Taiwanese study, the average quantity of alcohol intake in this group was only $175 \mathrm{~g}$ per week. We speculate that these Taiwanese HBV carriers were relatively protected from heavy alcohol drinking by their ALDH2 deficiencies.

\section{THE HYPOTHESIS}

We propose that $\mathrm{HBV}$ infection plays an important role in the evolution of the ALDH2*2 allele amongst Chinese Han. In the long-term HBV endemic in China, non- 
alcohol-drinking HBV carriers may have evolutionary advantages over alcohol-drinking HBV carriers. HBV carriers who drink less alcohol or do not drink any alcohol have better chances to survive than HBV carriers who drink more alcohol. We further suggest that through the natural selection of liver diseases, the ALDH2*2 allele becomes the adaptive gene amongst Chinese Han.

The fact that the chance of death from liver cancer is about threefold higher for male HBV carriers than is the case for female $(17,22)$ has important implications in our hypothesis. While the premature death from liver diseases of males affects the distribution of ALDH2 genes, female HBV carriers, who have a better chance to survive, warrant passing the virus to the next generation through perinatal transmission (18). For certain ALDH2active, alcohol-drinking, and HBV-infected families, the clustering premature death from liver cancer or cirrhosis may facilitate the process of natural selection.

The transmission of HBV infection and the diffusion of the ALDH2*2 allele, however, might follow different routes of travel and migration in history. There is good geographical correlation between HBV prevalence and ALDH2 deficiency within China, Asia, and, for that matter, the rest of the world. Populations that carry the ALDH2*2 alleles all reside within high-HBV-prevalence areas. Although HBV can be more easily transmitted by human contact, the spread of the ALDH2-mutated genes depends solely upon inter-racial marriage or migration. African people, for example, reflect a high HBV prevalence but virtually no ALDH2 deficiency, possibly because the mutant ALDH2*2 allele may never have been introduced to their gene pools.

This hypothesis is also supported by the strong synergistic interaction of alcohol drinking and HBV infection upon the risk of hepatocellular carcinoma found in non-ALDH2-deficient populations (24).

\section{TESTING THE HYPOTHESIS}

Our hypothesis predicts that HBV carriers with ALDH2*2 alleles demonstrate a lower risk of liver cirrhosis or liver cancer than is the case for HBV carriers who do not exhibit the ALDH2*2 alleles. In order to confirm or negate this hypothesis, one may design case-control studies to test if the prevalence of the ALDH2*2 allele is lower amongst cases of hepatocellular carcinoma or liver cirrhosis than is the case amongst controls. Alternatively, one may design cohort studies to test if HBV carriers revealing ALDH2*2 alleles are protected from premature death from liver diseases.

A third way to test the hypothesis is to trace the historical routes of HBV transmission and human migration through anthropological and archeological studies. Since Livingstone's early anthropological study of sickle-cell anemia and malaria in West Africa (26), the advancement of genomic science has made such studies much more feasible.

While vaccination has been a successful human approach to the eradication of $\mathrm{HBV}$ in modern Taiwan $(19,27)$, the evolution of ALDH2 deficiency and the emergence of a low-alcohol-drinking culture might be 'Mother Nature's' effort to prevent excessive premature death from liver disease in ancient China. We are yet to demonstrate that the HBV has accomplished something that the first emperor of the Xia dynasty had tried, but failed, to do-namely, keeping his Chinese descendants from the overindulgence of alcohol drinking.

\section{ACKNOWLEDGMENT}

The authors are indebted to Jung-Der Wang, MD, ScD, for his critical review of the manuscript and many helpful comments.

\section{REFERENCES}

1. Chang K. C. Ancient China. In: K. C. Chang (ed). Food in Chinese Culture: Anthropological and Historical Perspectives. New Haven: Yale University Press, 1977: 23-52.

2. Schafer E. H. T. In: K. C. Chang (ed). Food in Chinese Culture: Anthropological and Historical Perspectives. New Haven: Yale University Press, 1977: 85-140.

3. Harrell S. Normal and deviant drinking in rural Taiwan. In: A. Kleinman, T. Y. Lin (eds). Normal and Abnormal Behavior in Chinese Culture. Boston: Kluwer, 1980: 49-59.

4. Li H. Z., Rosenblood L. Exploring factors influencing alcohol consumption patterns among Chinese and Caucasians. J Stud Alcohol 1994; 554: 427-433.

5. Cheung Y. W. Beyond liver and culture: a review of theories and research in drinking among Chinese North America. Int J Addict 1993; 28: 1497-1513.

6. Goedde H. W., Harada S., Agarwal D. P. Racial differences in alcohol sensitivity: A new hypothesis. Hum Genet 1979; 51: 331-334.

7. Agarwal D. P., Goedde H. W. The role of alcohol metabolizing enzymes in alcohol sensitivity, alcoholdrinking habits, and incidence of alcoholism in Orientals. In: T. N. Palmer (ed). The Molecular Pathology of Alcoholism. New York: Oxford University Press, 1991: 211-237.

8. Wall T. L., Thomasson H. R., Ehlers C. L. Investigatorobserved alcohol-induced flushing but not self-report of flushing is a valid predictor of ALDH2 genotype. J Stud Alcohol 1996; 57: 267-272.

9. Chao Y. C., Liou S. R., Chung Y. Y. et al. Polymorphism of alcohol dehydrogenase genes and alcoholic cirrhosis in Chinese patients. Hepatology 1994; 19: 360-366.

10. Chen W. J., Loh E. W., Hsu Y. P. P., Chen C. C., Yu J. M., Cheng A. T. A. Alcohol-metabolising genes and alcoholism among Taiwanese Han men: Independent effect of ADH2, ADH3 and ALDH2. Br J Psychiat 1996; 168: 762-767.

11. Chen W. J., Loh E. W., Hsu Y. P. P., Cheng A. T. A. Alcohol dehydrogenase and aldehyde dehygrogenase genotypes and alcoholism among Taiwanese aborigines. Biol Psychiat 1997; 41: 703-709. 
12. Shen Y. C., Fan J. H., Edenberg H. J. et al. Polymorphism of $\mathrm{ADH}$ and $\mathrm{ALDH}$ genes among four ethnic groups in China and effects upon the risk for alcoholism. Alcohol Clin Exp Res 1997; 21: 1272-1277.

13. Doll R., Peto R., Hall E., Wheatly K., Gray R. Mortality in relation to consumption of alcohol: 13 years' observations on male British doctors. BMJ 1994; 3309: 911-918.

14. Thun M. J., Peto R., Lopez A. D. et al. Alcohol consumption and mortality among middle-aged and elderly US adults. $N$ Engl J Med 1997; 337: 1705-1714.

15. Anonymous. Leading causes of death, Taiwan Area, 19521997. Taiwan Health Information System, Department of Health, Taiwan, 2001. Available from http:// www.doh.gov.tw/english/statistics/.

16. Chen D. S., Sung J. L. Hepatitis B virus infection and chronic liver disease in Taiwan. Acta Hepato-Gastroenterol 1997; 25: 423-443.

17. Beasley R. P. Hepatitis B virus as the etiologic agent in hepatocellular carcinoma-Epidemiologic considerations. Hepatology 1982; 2: 21S-26S.

18. Stevens C. E., Beasley R. P., Tsui J., Lee W. C. Vertical transmission of hepatitis B antigen in Taiwan. N Engl J Med 1975; 292: 771-774.

19. Chang M. H., Chen C. J., Lai M. S. et al. Universal hepatitis B vaccination in Taiwan and the incidence of hepatocellular carcinoma in children. N Engl J Med 1997; 336: 1855-1859.
20. Beasley R. P., Lin C. C., Chien C. S., Chen C. J., Hwang L. Y. Geographic distribution of HBsAg carriers in China. Hepatology 1982; 25: 553-556.

21. Sung J. L., Chen D. S. Geographical distribution of the subtypes of hepatitis B surface antigen in the Chinese. Gastroenterol Jpn 1977; 12: 58-63.

22. Bosch F. X., Ribes J., Borras J. Epidemiology of primary liver cancer. Semin Liver Dis 1999; 19: 271-285.

23. Chen P. J., Chen D. S. Hepatitis B virus infection and hepatocellular carcinoma: Molecular genetics and clinical perspectives. Semin Liver Dis 1999; 19: 253-261.

24. Donato F., Tagger A., Chiesa R. et al. Hepatitis B and C virus infection, alcohol drinking, and hepatocellular carcinoma: A case-control study in Italy. Hepatology 1997; 26: 579-584.

25. Yu M. W., Hsu F. C., Sheen I. S. et al. Prospective study of hepatocellular carcinoma and liver cirrhosis in asymptomatic chronic hepatitis B virus carriers. $A m J$ Epidemiol 1997; 145: 1039-1047.

26. Livingstone F. B. Anthropological implications of sickle cell gene distribution in West Africa. Am Anthropol 1958; 60: 533-562

27. Chen H. L., Chang M. H., Ni Y. H. et al. Seroepidemiology for hepatitis $B$ virus infection in children: ten years of mass vaccination in Taiwan. JAMA 1996; 276 906-908. 